\title{
Un análisis comparativo de los sistemas de formación profesional en Extremo Oriente: los casos de China, Taiwán, Corea del Sur y Japón
}

\author{
Ana Beatriz Hernández Lara \\ Universidad Rovira i Virgili \\ anabeatriz.hernandez@urv.cat \\ Rosalía Cascón Pereira \\ Universitat Rovira i Virgili \\ rosalia.cascon@urv.cat
}

Resumen: Extremo Oriente destaca actualmente por sus indicadores sobre eficiencia y valor de la formación, según organismos internacionales como la Organización para la Cooperación y el Desarrollo Económicos (OCDE). Este artículo describirá las principales características de algunos sistemas de formación profesional de Extremo Oriente con el fin de proceder a un análisis comparativo de estos sistemas. En particular, se describirán y analizarán comparativamente los casos de China, Taiwán, Corea del Sur y Japón, que se han seleccionado como ejemplos significativos de esta área geográfica por sus sistemas de educación $y$, de forma más específica, por el desarrollo de su formación profesional. Con ello, se pretende contribuir a mejorar el conocimiento sobre los sistemas de formación profesional de esta región geográfica y analizar su repercusión en la formación de la fuerza laboral para extraer lecciones que puedan extrapolarse $y$ mejorar el sistema de formación profesional en nuestro entorno más cercano.

Palabras clave: sistema de educación; formación profesional; estudio comparativo; Extremo Oriente. 
A comparative analysis of the vocational education and training systems in the Far East: The cases of China, Taiwan, South Korea and Japan

\begin{abstract}
Far East currently stands out because of its indicators of efficiency and value in education according to international institutions like the Organization for the Cooperation and the Economic Development. This article describes the main characteristics of some of the vocational education and training (VET) systems developed in the Far East, with the aim of analyzing them comparatively. In particular, the cases of China, Taiwan, South Korea and Japan are described and analysed as relevant examples of this geographic area when analyzing their educational systems, and more specifically their VET systems. Therefore, we aim to contribute in improving the knowledge on the VET systems of this geographic area and in analyzing their impact on the formation and training of the workforce, in order to extract lessons that could also be applied to improve our educational system.
\end{abstract}

Keywords: Education systems, Vocational education and training, Comparative study, Far East. 


\section{Introducción}

La región de Extremo Oriente a la que se refiere el presente artículo es una región amplia y muy heterogénea. Constituye una región muy poblada, que acoge aproximadamente un $61 \%$ de toda la población mundial (CESCAP, 2014), y con una gran diversidad en los ámbitos demográfico, cultural y económico, lo que de alguna manera también conduce y explica cuáles pueden ser sus fortalezas y principales retos en lo que se refiere al ámbito educativo.

En el contexto asiático, y de forma más concreta en Extremo Oriente, los cambios económicos y sociales de las últimas décadas se han trasladado a los modelos educativos. En toda la región se entiende como fundamental conseguir una fuerza de trabajo con unas habilidades apropiadas y una buena formación para lograr prosperidad, crecimiento y competitividad. Algunas de estas habilidades se logran a través de la educación general, pero otras, las habilidades ocupacionales más específicas, requieren de una formación especializada que los sistemas educativos deben articular, si se desea conseguir una fuerza de trabajo profesional con un perfil competencial elevado. Así pues, son los sistemas de formación profesional (FP) los que, también en el contexto asiático, se han encargado tradicionalmente de proporcionar la formación ocupacional específica al dotar a los estudiantes del perfil de competencias y habilidades demandado por un mercado laboral cambiante.

En los últimos años, los sistemas de formación profesional han sido objeto de un intenso escrutinio y revisión para determinar si son capaces de desarrollar este perfil competencial en los estudiantes. Como consecuencia, se ha producido un movimiento reformista para dotar de una mayor calidad y uniformidad a los sistemas de formación profesional, lo que ha motivado un movimiento en los ámbitos institucional y gubernamental para ayudar a los estados en este propósito. Un ejemplo lo constituye la iniciativa lanzada en 2007 Learning for jobs, de la $\operatorname{OCDE}$ (2010), que plantea una serie de directrices para guiar la reforma de los sistemas de formación profesional en algunos países. Entre estos países, destacan algunos de Extremo Oriente, como Corea del Sur, China o Japón, entre otros.

Para conseguir el objetivo de este trabajo, centrado en sintetizar las principales características de los sistemas de formación profesional en algunos países de Extremo Oriente, destacar sus similitudes y diferencias, y extrapolar lecciones útiles a otros sistemas de formación profesional, este se ha estructurado en los siguientes apartados. La primera sección describirá el funcionamiento y las particularidades de los sistemas de formación profesional de China, Taiwán, Corea del Sur y Japón. La segunda sección destacará las principales similitudes y diferencias entre los sistemas de formación profesional de Extremo Oriente anali- 
zados. La última parte concluirá con el análisis de los principales puntos fuertes de los modelos de FP analizados con el fin de extraer lecciones aplicables a otros sistemas educativos como el español.

\section{Descripción de los sistemas de formación profesional en Extremo Oriente}

\subsection{China}

China se ha convertido en los últimos años en una de las economías más potentes a nivel mundial, puesto que ha crecido alrededor de un $8 \%$ y un $9 \%$ anual y alcanzó un PIB en dólares corrientes de 10,36 trillones en 2014 (Banco Mundial, 2015). Incluso en los peores momentos de la recesión global reciente, si bien su crecimiento se ha reducido, ha seguido siendo robusto, y sus previsiones de crecimiento para 2016 se sitúan en torno al 6,7 \% (OCDE, 2015). Como consecuencia de la mejora en las condiciones económicas del país, el nivel de vida de su población, en términos generales, también ha mejorado enormemente, lo que se traduce en el ámbito de la educación en una universalización de la educación primaria y de los niveles más básicos de educación secundaria, unas tasas cada vez más altas de estudiantes en los niveles más altos de educación secundaria y también una participación significativamente creciente en la educación universitaria.

Dentro del sistema educativo de China, la FP ocupa un lugar importante y, además, su relevancia ha ido creciendo en los últimos años dada su crucial repercusión en la mejora del nivel de empleo y la formación y el entrenamiento de la mano de obra cualificada, que se considera necesaria para seguir potenciando la economía china (Unesco-Unevoc, 2013).

La legislación aplicable al sistema de formación profesional consiste fundamentalmente en la Ley de Formación Profesional (Vocational Education Lax) de la República Popular China del año 1996. Esta ley intentó acelerar la reforma y el desarrollo del sistema de formación profesional en China y lo regula en sus distintos niveles. Otros documentos que regulan aspectos específicos del sistema de formación profesional en China son la Ley Laboral de 1994, la Ley de Profesorado de 1994, la Ley de Educación Obligatoria adoptada en 1986, la Ley de Promoción de la Educación Privada de 2002 y el Plan Nacional para la Reforma y el Desarrollo Educativo a medio y largo plazo, que abarca el periodo comprendido entre 2010 y 2020 (Unesco-Unevoc, 2013).

$\mathrm{El}$ informe Learning for jobs. Reviews of vocational education and training. Options for China (OCDE, 2010) desarrolla una descripción detallada de las principales características del sistema de formación profesional en China, que puede 
resumirse en los siguientes aspectos. En China, la FP tiene lugar en cuatro contextos fundamentales:

- Escuelas de educación secundaria de nivel inferior (residual).

- Escuelas de educación secundaria de nivel superior.

+ Educación universitaria o de tercer nivel.

- Enseñanza de adultos y formación en empresas.

En la figura 1 puede apreciarse un esquema de cómo se integra la FP en el sistema educativo chino.

Figura 1. Esquema del sistema educativo chino.

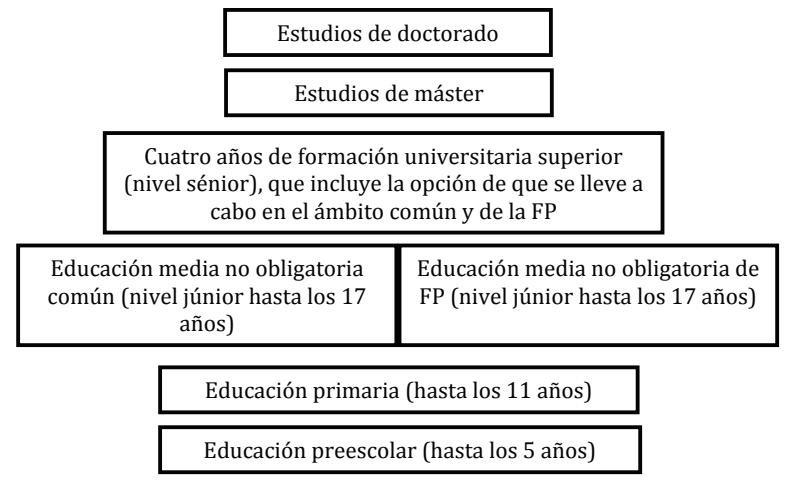

Fuenteः Esquema adaptado de Unesco-IBE (2011): World data on education: people's Republic of China.

En China son obligatorios nueve años de escolarización a tiempo completo. Esta etapa se divide en la mayor parte de las provincias en seis años de educación primaria y tres años de educación secundaria de nivel inferior o júnior. El sistema de cinco más cuatro también se aplica en algunas provincias. La edad de entrada en el sistema obligatorio de educación son los 6 años. En la actualidad, esta formación obligatoria es prácticamente universal. En esta etapa, la educación secundaria de nivel inferior abarca tanto la educación genérica como la FP júnior. Las escuelas de educación secundaria de nivel inferior están en declive (con menos de $1 \%$ del total de estudiantes) (OCDE, 2010). Han desaparecido prácticamente en las áreas urbanas y solo se mantienen en las áreas rurales, donde la economía está menos desarrollada y se proporcionan habilidades básicas relacionadas con la agricultura y los trabajos artesanales.

Aproximadamente tres cuartas partes de los estudiantes continúan, una vez cursada la educación obligatoria, con la educación secundaria de nivel superior, que se divide, a su vez, en formación secundaria genérica o profesional (FP). Un 
sistema de evaluación nacional determina el acceso de los estudiantes a ambos itinerarios formativos. $\mathrm{Al}$ respecto, cabe señalar que la educación secundaria genérica, cuya duración es de tres años, requiere una mayor puntuación en el examen nacional para acceder a ella. A partir del año 2002, se ha instaurado un sistema en el que al final del programa formativo, el estudiante debe pasar una evaluación de habilidad general, administrada por las autoridades provinciales y que posibilita el poder graduarse en esta etapa. En el caso de la FP sénior o de nivel superior son las escuelas especializadas, las escuelas técnicas o para trabajadores cualificados y los institutos de FP sénior los que ofertan estos programas formativos, cuya duración oscila entre los tres y cuatro años (Unesco-IBE, 2011).

El objetivo de la FP de nivel superior es formar a la fuerza de trabajo y permitirle la adquisición de habilidades prácticas. En cuanto al itinerario de acceso, los graduados de los centros de FP júniores son los que pueden acceder a los centros que ofertan esta educación superior. Por su parte, la formación vocacional ofrecida en el ámbito universitario acoge a estudiantes de FP sénior y a estudiantes de la secundaria genérica, y se desarrolla en programas de dos o tres años. Las instituciones que imparten este tipo de formación son principalmente instituciones tecnológicas de FP superior y universidades que proporcionan estudios especializados de FP o vocacionales. La FP a este nivel hace hincapié en la formación práctica o experiencial orientada al empleo para formar talentos altamente especializados en temas técnicos y de gestión.

Existe la opción de continuar la FP con estudios universitarios tradicionales. Para ello, una vía, aunque no la única, es superar un examen nacional, aunque hay cuotas que determinan el número máximo de estudiantes que pueden acceder a la universidad por esta vía. El prestigio que en los últimos años ha caracterizado a los estudios universitarios ha provocado un aumento en los estudiantes universitarios que acceden a la universidad utilizando cualquier vía, lo que incluye entre las distintas opciones también los estudios de FP como paso previo antes de iniciar la formación universitaria, aunque no es la opción más frecuente (OCDE, 2010).

De acuerdo con la Ley de Educación, son el Estado y los gobiernos locales los encargados de guiar y administrar la educación en sus distintos niveles para garantizar una división sostenible de las responsabilidades (Unesco-IBE, 2011). Los organismos que tienen la máxima responsabilidad en relación con la FP en China son dos: el Ministerio de Educación, que se encarga de la formación genérica, más técnica y vocacional, y el Ministerio de Recursos Humanos y Seguridad Social, que pone más atención en el entrenamiento de habilidades orientadas al empleo. Localmente, ambos ministerios tienen departamentos de educación y 
de recursos humanos y de seguridad social que se encargan de las rutinas diarias involucradas en la administración de la FP, como sería el caso de la gestión presupuestaria o del personal.

Aun así, lo más habitual es que la FP dependa de instituciones municipales o territoriales, como ayuntamientos u organismos similares. En otras ocasiones, las provincias o agencias gubernamentales, como sería el caso de ciertas oficinas ministeriales, se encargan de los centros de FP que imparten educación técnica en sus áreas de especialidad. Algunos ejemplos son el Bureau de Agricultura o la Comisión de Transportes de Pekín (OCDE, 2010).

Por otro lado, las empresas también están involucradas en la provisión de este tipo de formación, sobre todo dirigido a sus propios empleados. Así pues, las empresas pueden establecer sus escuelas de FP de forma autónoma, o bien en cooperación con otras empresas. De igual modo, las empresas pueden confiarle esta tarea sobre la formación específica de sus trabajadores o de sus futuros trabajadores a escuelas de FP con las que firmen convenios (Ministerio de Educación, 1996).

Además de las empresas, las necesidades del entorno fuertemente cambiante han hecho proliferar escuelas no oficiales de FP. Estas escuelas tienen una gran autonomía para adoptar decisiones y proporcionan fundamentalmente cursos de corta duración que facilitan una formación muy dirigida y específica para cubrir determinadas habilidades demandadas por el mercado. Aunque estos centros no dependen del Estado, necesitan una licencia emitida por el departamento de educación que les corresponda, y se dividen en organizaciones sin ánimo de lucro, como asociaciones profesionales o sociales, sindicatos, etc., y organizaciones comerciales.

El desarrollo curricular de los estudiantes de formación profesional se divide en tres partes. Una tercera parte de las habilidades la determina el Ministerio de Educación; otra tercera parte, también en el ámbito nacional, define las competencias asociadas a cada ocupación particular; y el último tercio depende de las necesidades locales y se determina en el ámbito de centro.

En total hay 270 especialidades de FP en todo el territorio nacional, que se agrupan en 13 ramas (OCDE, 2010), que incluyen, entre otras: agricultura y bosques, recursos naturales y medioambiente, energía, ingeniería civil e hidráulica, manufactura, transporte, tecnologías de la información, medicina y salud, negocios, comercio y turismo, finanzas y economía, cultura, arte y deportes, relaciones públicas y asuntos sociales.

El objetivo del Gobierno chino es que aproximadamente la mitad de los estudiantes opten por la educación secundaria genérica, mientras que la otra mitad 
cursen estudios de FP (OCDE, 2010). Sin embargo, cada vez es más evidente la importancia de la educación universitaria o de tercer nivel, que se está convirtiendo en una aspiración para padres y estudiantes, lo que influye en la elección de la educación secundaria genérica o común como la opción más atractiva, por encima de la FP. Esta realidad constituye un acicate para profundizar en las reformas del sistema de formación profesional, de modo que la formación ofrecida por el sistema permita mejorar el perfil competencial del estudiante, lo vincule de forma más evidente con las necesidades de la industria, y que el egresado del sistema de formación profesional se convierta en una opción de primer nivel para los empleadores, es decir, que se constituya como una opción formativa también atractiva para los propios estudiantes.

\subsection{Taiwán}

Taiwán, que es el nombre por el que se reconoce normalmente a la República de China, constituye un Estado soberano del este de Asia. Entre las particularidades de este territorio, que lo alejan de la China continental, destacan algunos aspectos en los ámbitos geográfico y político: la República de China se constituye como una república democrática y geográficamente comprende la isla de Taiwán, que representa prácticamente el 99 \% de su territorio, la isla de Pescadores y conjuntos de archipiélagos, como los de Quemoy y Matsu, principalmente.

Económicamente esta región ha experimentado un avance reseñable, que le ha valido el reconocimiento de «desarrollo milagroso» (Yao y Tsang, 2012). No obstante, la actual vitalidad de Taiwán no se entiende si no se tienen en consideración las consecuencias conjuntas de su sistema democrático y su economía de mercado. Estos cambios justifican los datos de sus principales indicadores económicos con un PIB nominal en 2013 de más de 489 billones de dólares, que ha ido creciendo en los últimos años, desde 2010, de forma ininterrumpida, hasta crecer en 2013 un 2,09\% y en 2014 un 3,7 \%, lo que representa un PIB per cápita ese año de 20952 dólares (The Republic of China yearbook, 2014).

Estas particularidades de Taiwán en los ámbitos político, cultural y económico afectan también a su sistema educativo y a su sistema de formación profesional, como parte del mismo.

El país cuenta con tres niveles administrativos: el nacional, el provincial y el municipal, y el Ministerio de Educación es el responsable del desarrollo y la implementación de las políticas educativas en todos los niveles. El sistema educativo taiwanés, en general, es un sistema centralizado que sigue el modelo anglosajón, especialmente el establecido en Norteamérica. Sin embargo, aunque tradicionalmente ha existido esta centralización y el Gobierno ha sido el responsable de toda 
la organización y el establecimiento de estándares en la educación, en la actualidad, el sistema está evolucionando y ya no está tan centralizado, deja más espacio para la diversidad y la flexibilidad (Ministry of Education, 2012). Un esquema de su estructura puede apreciarse en la figura 2.

Figura 2. Esquema del sistema educativo taiwanés.

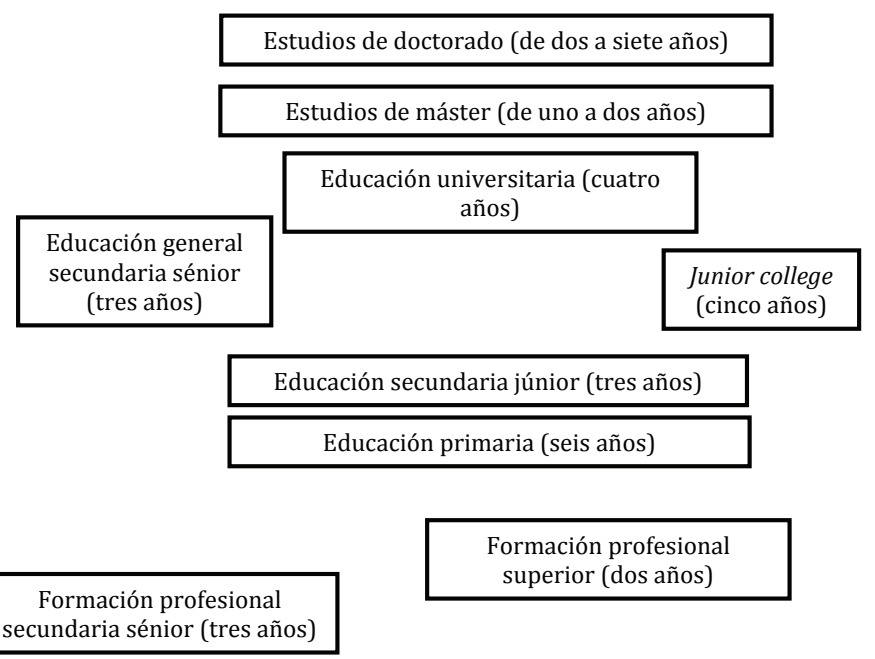

Fuente: Esquema adaptado de Education System Taiwan (2011).

La educación obligatoria cubre un periodo de nueve años y se extiende por las etapas de educación primaria y educación secundaria júnior o intermedia. El Gobierno estableció una reforma para ampliar este periodo de educación obligatoria y que a partir de 2014 fuera de 12 años en vez de nueve (Ministry of Education, 2012). En el sistema educativo actual, por encima del nivel de educación secundaria júnior se abren dos vías formativas: la educación secundaria genérica sénior y la FP. Una mirada más específica al modelo de FP permite diferenciar entre el nivel intermedio y el superior. El intermedio incluye programas de habilidades y oficios júniores, mientras que el nivel superior comprende programas profesionales más completos e intensivos.

El número de estudiantes en Taiwán que deciden optar por la FP supera el $60 \%$ y está por encima del porcentaje de estudiantes que optan por la formación secundaria genérica, lo que es una particularidad de este modelo que no se comparte en el resto del mundo (Ministry of Education, 2014). De hecho, el desarrollo económico de Taiwán, que comienza a hacerse patente a partir de la década de los cincuenta, se debe en buena medida al establecimiento y desarrollo de su sistema de formación profesional (Ministry of Education, 2012). La evolución conjunta de ambas varia- 
bles puede apreciarse en la tabla 1. Este desarrollo económico vino motivado por la actuación del Gobierno, la flexibilidad y fortaleza de sus pequeñas y medianas empresas, y la fuerza de trabajo de esta región, que ha crecido de forma muy relevante en las últimas décadas (ICDF, 2010). Cuando se inició el desarrollo económico de la región, había una gran necesidad de mano de obra cualificada, por lo que se instauró una fuerte demanda de instituciones de formación técnica. Así, en 1976 la creación de estas instituciones de formación se convirtió en el principal objetivo del sistema educativo y se formuló como «la necesidad de orientar el sistema hacia la enseñanza de ciencia aplicada y tecnología para formar personal profesional con habilidades prácticas» (ICDF, 2010).

\section{Tabla 1. Comparativa entre el desarrollo económico} y el desarrollo de la FP en Taiwán.

\begin{tabular}{|c|c|c|}
\hline Años & Desarrollo económico & Desarrollo de la FP \\
\hline $\begin{array}{l}\text { Década } \\
\text { de } 1950\end{array}$ & $\begin{array}{l}\text { - Reforma de la tierra. } \\
\text { - Incremento de la productividad } \\
\text { agrícola. } \\
\text { - Desarrollo de industrias de } \\
\text { bienes básicos intensivas en } \\
\text { mano de obra. }\end{array}$ & $\begin{array}{l}\text { - Educación en agricultura y comercio. } \\
\text { - Mayor atención a las escuelas de FP de nivel } \\
\text { sénior. }\end{array}$ \\
\hline $\begin{array}{l}\text { Década } \\
\text { de } 1960\end{array}$ & $\begin{array}{l}\text { - Expansión de los negocios de } \\
\text { importación-exportación. }\end{array}$ & $\begin{array}{l}\text { - Desarrollo de la FP industrial y comercial. } \\
\text { - Nueve años de educación obligatoria. } \\
\text { - Aumento de los programas de FP y del número } \\
\text { de centros y estudiantes. } \\
\text { - Inicio de los sistemas de cinco y dos años de } \\
\text { junior college. }\end{array}$ \\
\hline $\begin{array}{l}\text { Década } \\
\text { de } 1970\end{array}$ & $\begin{array}{l}\text { - Expansión de la construcción. } \\
\text { - Expansión de las industrias } \\
\text { intensivas en capital y tecnología. }\end{array}$ & $\begin{array}{l}\text { - Establecimiento de institutos tecnológicos. } \\
\text { - Mejora de la FP industrial y de la educación en } \\
\text { el junior college. }\end{array}$ \\
\hline $\begin{array}{l}\text { Década } \\
\text { de } 1980\end{array}$ & $\begin{array}{l}\text { - Desarrollo de industrias de alta } \\
\text { tecnología. } \\
\text { - Desarrollo de la industria } \\
\text { petroquímica. }\end{array}$ & $\begin{array}{l}\text { - Mejora generalizada de la cantidad y la calidad } \\
\text { de la FP industrial y de la educación de nivel de } \\
\text { junior college. }\end{array}$ \\
\hline $\begin{array}{l}\text { Década } \\
\text { de } 1990\end{array}$ & $\begin{array}{l}\text { - Desarrollo de la economía del } \\
\text { conocimiento. } \\
\text { - Planificación del centro de } \\
\text { operaciones Asia-Pacífico. }\end{array}$ & $\begin{array}{l}\text { - Establecimiento masivo de escuelas de for- } \\
\text { mación secundaria. } \\
\text { - Aumento de los colleges de tecnología. } \\
\text { - Transformación de los junior colleges prestigi- } \\
\text { osos en colleges tecnológicos. } \\
\text { - Transformación de los colleges tecnológicos } \\
\text { prestigiosos en universidades de ciencia y tec- } \\
\text { nología. }\end{array}$ \\
\hline $\begin{array}{l}\text { Década } \\
\text { de } 2000\end{array}$ & $\begin{array}{l}\text { - Desarrollo de nuevos sectores } \\
\text { industriales. }\end{array}$ & - Maximización e internacionalización de la FP. \\
\hline $\begin{array}{l}\text { Década } \\
\text { de } 2010\end{array}$ & - Desarrollo industrial. & $\begin{array}{l}\text { - Mayor atención al encaje entre las demandas de } \\
\text { la fuerza de trabajo industrial y las aptitudes de } \\
\text { los estudiantes. }\end{array}$ \\
\hline
\end{tabular}

Fuente: Tabla adaptada del Ministry of Education (2014). 
En la década de los cincuenta, las actividades económicas más importantes estaban relacionadas con la agricultura, las industrias básicas y el comercio. Estas actividades comerciales se fueron ampliando y se incorporaron actividades de comercio exterior a partir de la década de los sesenta. Para cubrir la formación que demandaban las empresas para su mano de obra, el Gobierno puso en marcha un número cada vez más elevado de centros de FP en los niveles de primaria y secundaria. Cuando comenzó la producción industrial en masa, las demandas de personal cualificado a nivel técnico se dispararon, pero también las de formación específica en temas comerciales, de administración de empresas y marketing, lo que se tradujo en un aumento de centros que impartían este tipo de FP. Por este motivo, el ámbito primordial de la FP sénior en ese momento eran los estudios agrarios y de administración de empresas. En la década de los noventa, con el surgimiento de la revolución tecnológica, se produjeron importantes cambios en la estructura industrial. Los estudiantes que hasta ese momento habían cursado estudios de FP empezaron a mostrar una mayor inclinación por desarrollar estudios universitarios superiores, a lo que el Gobierno respondió permitiendo que las escuelas técnicas se transformaran en institutos tecnológicos para que pudieran llevarse a cabo programas de dos años de formación superior en tecnología, y se implementó también una política de FP ampliable durante este periodo.

El sistema de formación profesional en Taiwán ha sido objeto de sucesivas reformas. De hecho, no se trata de una formación que tenga un excesivo recorrido histórico, lo que hace que el modelo esté siendo objeto aún de revisión. El Ministerio de Educación lanzó un proyecto de reforma en el año 2009 que se desarrollaba en tres fases y que era paralelo a las reformas que se iniciaron en el ámbito político-económico. Las reformas económica y educativa fueron de la mano y esto es lo que explica que el sistema de formación profesional esté tan vinculado en el desarrollo económico del país. En la actualidad, se está llevando a cabo la fase 2 (2013-2017), cuyas principales metas son las siguientesः asegurar que los graduados de FP encuentren un empleo de forma inmediata, proporcionar una mano de obra con una formación de alta calidad según las demandas de la industria y reformar la imagen que tiene la sociedad de la FP. Todo ello con la intención de hacer que la FP sea más competitiva respecto a otros tipos de formación alternativos. Entre los objetivos incluidos en la reforma también destaca que la formación secundaria, tanto la genérica como la de FP, esté exenta del pago de matrículas y de la presentación de una evaluación o un examen que determine la admisión o no a estos programas formativos, lo que permite una FP aún más abierta e inclusiva para todo tipo de estudiantes (Ministry of Education, 2012 y 2014). De hecho, se pretende que exista un proceso de evaluación único para determinar la entrada de estudiantes, que se gestione desde lo que se denomina 
el Testing Center for Technological and Vocational Education, y que permita que los estudiantes solo pasen un único examen que pueda usarse independientemente del centro o la materia a la que quiera accederse.

En cuanto a la financiación de los estudios de FP, y con la intención de apoyar la reforma de enseñanza obligatoria durante 12 años, los institutos de FP, incluidos, además, los primeros años de college, se incorporaron dentro de un programa de apoyo financiero para que todos los estudiantes con ingresos bajos pudieran cursar estudios de FP de nivel superior, de modo que todos los gastos los soporta el Gobierno (Ministry of Education, 2012). Del mismo modo, los estudiantes con algún tipo de discapacidad también cuentan con ayuda financiera específica desde el año 2005 para que puedan superar sus limitaciones y llevar a cabo sus estudios de FP (Ministry of Education, 2012).

En cuanto a la estructura administrativa del sistema educativo en Taiwán, es el Departamento de Educación Vocacional y Tecnológica (DTVE), en dependencia directa del Ministerio de Educación, el que se ocupa de todos los aspectos relacionados con la FP en el ámbito nacional. El DTVE también se ocupa y supervisa todas las universidades de ciencia y tecnología, y los colleges de tecnología júniores y séniores. En Taiwán existen cinco municipalidades especiales, y cada una de ellas tiene su propio bureau de educación que se encarga de la FP de nivel intermedio dentro de su jurisdicción. Cada ciudad o county tiene igualmente su propio bureau de educación para ocuparse de las escuelas de FP sénior, así como de los programas formativos de habilidades técnicas dentro de su jurisdicción.

A pesar de que la descripción del sistema de formación profesional está muy centrada en la formación pública y las regulaciones que el Gobierno realiza sobre este modelo, lo cierto es que en Taiwán las instituciones privadas involucradas en la FP son muy importantes. Así, en 2013 el porcentaje de estudiantes que cursaban estudios de FP sénior en centros privados ascendía al 62,4 \%, y en el caso de los estudiantes de FP en junior colleges, el porcentaje creció hasta el 80,2 \%. Para los estudiantes, una de las mayores ventajas de realizar esta formación en una institución privada tiene que ver con los fuertes vínculos que existen entre estos centros y el tejido empresarial e industrial, lo que permite que la formación recibida tenga una orientación práctica muy marcada.

A continuación, se llevará a cabo una descripción más detallada de la formación que se imparte en los diferentes niveles del modelo de FP (Ministry of Education, 2012 y 2014).

En el nivel intermedio de FP, los programas formativos se imparten en los institutos júniores y están abiertos a los que terminan la formación obligatoria, que tienen aptitudes e inclinación hacia algún oficio. Están diseñados para que 
los estudiantes tengan tiempo para explorar qué tipo de carrera profesional quieren llevar a cabo.

Los grupos profesionales que se imparten en institutos séniores son programas de tres años y admiten a estudiantes graduados en el instituto júnior o en niveles equivalentes. Estos programas se centran en la formación de habilidades prácticas y siguen un enfoque basado en el estudiante, ya que buscan su desarrollo profesional y tratan cada caso de forma individualizada. Estos cursos están orientados a estudiantes que desean formarse en una profesión técnica y que tienen aptitudes para ello, y se les da oportunidades de empleo directo una vez diplomados, lo que les permite integrarse en el mundo laboral a las clases más desfavorecidas. Estos grupos profesionales se desarrollan en seis disciplinas: agricultura, industria, negocios y actividad comercial, actividades marinas y pesca, ciencia, y arte. La educación que se recibe es eminentemente práctica para que la persona diplomada se adecúe perfectamente a la industria desde el principio. Los estudiantes que eligen esta opción pueden entrar directamente al mundo laboral a través de un contrato por cuenta ajena o de la creación de su propia empresa, o pueden acceder a estudios universitarios superiores; en este caso pueden cursar programas de dos años de los junior colleges, los colleges de tecnología o las universidades de ciencia y tecnología.

Por su lado, el nivel superior de la FP en Taiwán se clasifica, a su vez, en dos estratos: el que se desarrolla en los junior colleges y el que tiene lugar en los colleges de tecnología o en las universidades de ciencia y tecnología. Los junior colleges pueden hacer formación de dos o cinco años. Entre sus especialidades formativas destacan las siguientes: administración de empresas, ingeniería, tecnología computacional, salud, agricultura, actividades marinas, etc. Los programas de ingeniería civil tienen una duración de un año más (Education System Taiwan, 2011). Para acceder a estudios universitarios superiores es necesario realizar un examen de acceso, aunque con tres años de experiencia laboral relevante acreditada podría accederse directamente a estudios de máster (Education System Taiwan, 2011). La instrucción en los estratos superiores de FP reconoce el valor del talento y la calidad profesional, por lo que desde el año 2010 se ha adoptado el sistema dual, que implica la incorporación de profesionales y expertos de la industria al sistema de formación profesional, para que de esta manera la interconexión entre la educación vocacional y la industria sea lo más fuerte y directa posible.

Como conclusión a este apartado, cabe afirmar que el sistema de formación profesional en Taiwán es un sistema comprehensivo que incluye formación en todos los niveles, desde los más básicos hasta los más elevados, los de formación 
universitaria doctoral, solo que en su vertiente más profesional, que es lo que caracteriza el modelo formativo de FP.

\subsection{Corea del Sur}

La expansión experimentada por el sistema educativo coreano es excepcional y ha desempeñado un papel crucial en el desarrollo económico de este país y en el crecimiento de su productividad. Los principales indicadores económicos sitúan a Corea como una potencia económica, con un PIB per cápita de 34356 dólares en 2014, y un crecimiento del PIB ininterrumpido desde 2009, que ha alcanzado su pico más alto en estos últimos años: en 2010 con un 6,5 \% y su previsión para 2016 asciende a un 3,64 \% según indican los datos más actualizados de la OCDE. De hecho, la expansión económica coreana ha merecido la denominación de milagro económico y ha permitido una rápida transformación de su sociedad y economía. Este crecimiento comenzó en la década de los sesenta y se basaba en una política gubernamental y en unas estrategias empresariales que favorecían cambios rápidos y profundos para evolucionar desde una sociedad fundamentada en la agricultura a otra donde primaran la manufactura y los servicios. Estos cambios favorecieron un fuerte crecimiento de las exportaciones $y$, con el paso del tiempo, que se hiciera hincapié en sectores con un peso tecnológico muy elevado (Kuczera, Kis y Wurzburg, 2009).

La expansión económica de Corea del Sur se ha producido en paralelo a su desarrollo educativo, que también recibe la denominación de milagro educativo, como cabe observar por el hecho de que el porcentaje de población que al menos tiene educación de nivel de secundaria es el más alto de toda la OCDE. El 65 \% de los adultos jóvenes tiene formación de tercer nivel o universitaria, y también ocupa posiciones de cabeza en relación con los principales indicadores utilizados por el Programa Internacional de Evaluación de los Alumnos (PISA) (Jones, 2013).

Los logros educativos de Corea del Sur se deben en gran medida a sus características culturales, como su intensa disciplina y el trabajo ético de sus estudiantes, a quienes los animan tanto sus familias como el profesorado. Sin embargo, también existe una gran insatisfacción respecto al sistema educativo debido a algunas de las consecuencias que provoca, como unos elevados niveles de estrés y ansiedad entre los estudiantes y sus padres y el problema financiero que supone para las familias proporcionarles una buena educación a sus hijos, que puede terminar provocando una distribución no equitativa respecto a las oportunidades educativas (Jones, 2013).

A continuación, se presentará un resumen de la estructura del sistema educativo coreano y la posición que ocupa en este el sistema de formación profesional. 
El sistema educativo coreano se basa en un modelo $6+3+3+4$ (Park, 2011), que consiste en seis años de escuela elemental, tres años de junior high school, tres años de bigh school y cuatro años de educación universitaria, como se aprecia en la figura 3.

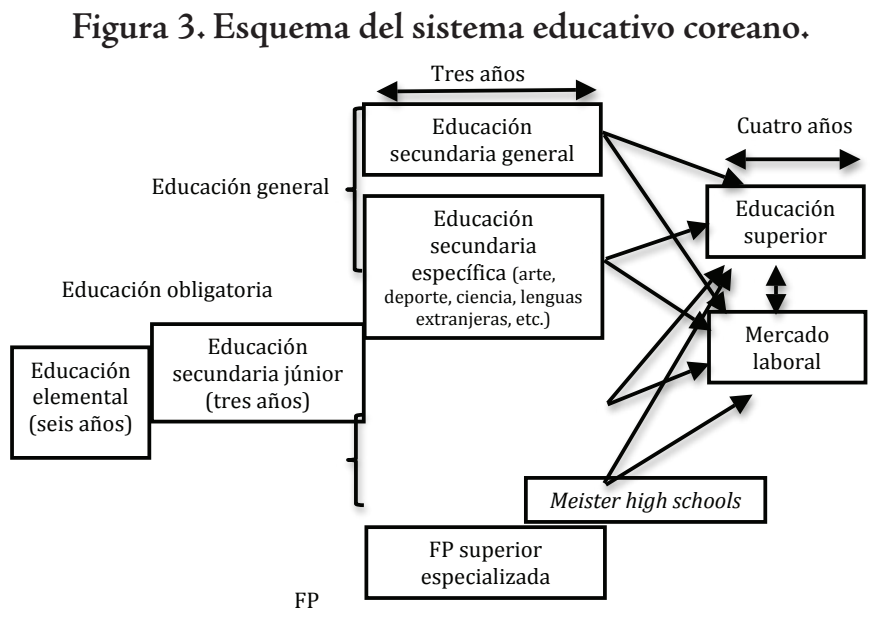

Fuente: Park (2011).

El modelo educativo en Corea del Sur está constituido por nueve años de educación obligatoria gratuita: los primeros seis años son de educación primaria y los siguientes tres tienen lugar en la middle school. El alto grado de escolarización hace de Corea del Sur uno de los pocos países en los que la graduación en niveles de educación secundaria es prácticamente universal, y una parte muy mayoritaria de los estudiantes continúan con estudios de tercer nivel o universitarios (Jones, 2013).

Los estudiantes se asignan de manera aleatoria entre las middle y las high schools (ambas privadas y públicas) en sus distritos a través de un sistema de lotería, que reemplaza el sistema anterior basado en un examen. Los centros de secundaria (high schools) pueden ser de dos tipos: los genéricos, que son los que preparan a los estudiantes para entrar posteriormente en la universidad, $y$ los vocacionales o de FP, que originalmente preparan a los estudiantes para que accedan al mercado laboral. Los programas que ofrecen estos centros difieren un poco dependiendo del público y la industria a la que se dirijan (Park, 2011). A estas dos modalidades, se le ha agregado en los últimos años una tercera modalidad de institución que proporciona este tipo de formación: las denominadas special purpose high schools o meister high schools, que se especializan en determinados ámbitos, como las ciencias naturales, las lenguas extranjeras, el arte y la educación física, y que ofrecen programas custo- 
mizados y directamente vinculados con las necesidades de la industria con la que se relaciona la formación que imparten (Park, 2011).

Una mención especial merece el tema de la formación privada en Corea del Sur, llevada a cabo en los denominados hagwons, que atraen a los mejores profesores y que pagan los salarios más atractivos. El uso de este sistema justifica buena parte del excelente desempeño de los estudiantes coreanos en los principales indicadores educativos, pero sobre todo en los niveles formativos más básicos, no tanto en la enseñanza universitaria (Jones, 2013). Su utilización está justificada, según la opinión de las familias, por las dificultades que encuentran los estudiantes para acceder a buenas universidades, que seleccionan a sus estudiantes en función de sus calificaciones (Chang, 2009).

La educación universitaria se ha expandido enormemente en las últimas décadas, hasta el punto de que se produce lo que se conoce como inflación educativa, cuyo efecto final es que los empleos que antes solían desempeñar estudiantes con educación secundaria ahora sean ocupados por graduados universitarios (Jones, 2013). A su vez, se reconocen dos tipologías de instituciones universitarias: los colleges, que son instituciones que imparten normalmente formación durante dos años y cuya atracción de estudiantes ha ido disminuyendo en los últimos años, ya que se considera que tienen un estatus inferior al de las instituciones que imparten formación universitaria superior, el otro tipo de institución, cuya duración en general es de cuatro años.

En lo que respecta a la financiación del sistema educativo coreano, el gasto total en educación de tercer nivel está bastante por encima de la media de la OCDE, aunque el sector público solo paga un $26 \%$ del total. El Estado se centra sobre todo en la financiación de la educación primaria y secundaria, mientras que las instituciones privadas son las que se encargan prácticamente de poner en marcha el sistema universitario en Corea del Sur (Jones, 2013). Los esquemas de financiación en ambos casos son muy distintos. Las universidades públicas o nacionales reciben fondos del Estado, que ascienden en torno a un $57 \%$, y el $23 \%$ restante corresponde a los ingresos de las matrículas que pagan los estudiantes. En cambio, en las universidades privadas, el $67 \%$ procede de las tasas y las matrículas.

En cuanto a la FP, en su modelo genérico, se estructura en programas que se llevan a cabo en cinco ámbitos distintos: agricultura, tecnología e ingeniería, comercio y negocios, ciencias marítimas y pesqueras, y economía doméstica.

La FP en Corea del Sur tiene un problema de popularidad que ha hecho que el número de estudiantes que optan por esta formación se reduzca. Además, los estudiantes que optan por la FP normalmente tienen un estatus socioeconómico 
más bajo que los estudiantes que optan por la vía educativa más genérica y su desempeño también es más bajo (Kuczera, Kis y Wurzburg, 2009).

A pesar de que durante mucho tiempo los centros de secundaria dedicados a la FP han jugado un papel crítico a la hora de suministrar la mano de obra cualificada necesaria para sostener el rápido crecimiento económico y el desarrollo de Corea del Sur, en los últimos tiempos se está revirtiendo este efecto, conforme la economía y el tejido productivo evolucionan hacia el desarrollo de otros sectores económicos, donde el uso de tecnologías y habilidades técnicas muy específicas va ganando terreno frente a las capacidades más básicas o genéricas (Park, 2011). La demanda de habilidades cognitivas es cada vez mayor, mientras que la de habilidades físicas o sensoriales está en declive (Hwang, 2007). Esto está jugando en contra de la FP tradicional, ya que la industria considera a los graduados de las vocational high schools como trabajadores con capacidades básicas, que solo son capaces de tener un desempeño adecuado en tareas no complejas, a diferencia de los estudiantes que han cursado otros niveles superiores de educación (Park, 2011). Este fenómeno explica la tendencia decreciente que muestran las vocational high schools desde el año 2010 y su escaso atractivo cuando se comparan con la educación secundaria general. El Gobierno coreano ha tratado de revertir esta tendencia cambiándole el nombre de vocational high schools a professional high schools (Kuczera, Kis y Wurzburg, 2009). De hecho, en la actualidad la FP ya no se considera como una etapa formativa final antes de acceder al mercado de trabajo, sino más bien, como una vía más para acceder a estudios superiores, aunque sea para acceder a los colleges (de carácter más técnico o vocationally-oriented) y no tanto a la universidad (Jones, 2013).

La gestión del sistema educativo en Corea del Sur descansa en dos ministerios: el Ministerio de Educación, para todo lo relacionado con la formación de habilidades estándares, y el Ministerio de Empleo y Trabajo, para todos los aspectos ocupacionales (Jones, 2013). La responsabilidad de la educación primaria y secundaria en las 16 provincias y ciudades metropolitanas descansa en consejos y oficinas de educación, que están separados de los gobiernos locales generales, lo que le otorga al sistema un nivel muy alto de descentralización (Jones, 2013). De acuerdo con la OCDE, el $46 \%$ de las decisiones en las middle schools coreanas se toman de forma autónoma por la escuela o dentro de un marco establecido por la autoridad superior, lo que está bastante cerca de la media de la OCDE. La mayor autonomía existe respecto a cómo se organiza la educación, y la más baja se produce en relación con la planificación y la estructura, y la gestión del personal. La propuesta que existe en estos momentos es seguir aumentando esta autonomía con el objeto de mejorar los resultados. 
Otra de las reformas para mejorar la educación secundaria está relacionada con tratar de lograr una mayor diversificación mejorando la competencia entre las escuelas o los centros y aumentando su diversidad. En el marco de esta reforma, hay medidas como el aumento del número de escuelas privadas autónomas, lo que mejora la autonomía curricular y de gestión, y una mayor incorporación al sistema de las meister high schools, que funcionan a semejanza del modelo alemán.

\subsection{Japón}

El difícil contexto natural de Japón, con escasez de recursos naturales, ha facilitado la construcción de una cultura cuyos valores realzan la importancia del desarrollo del capital humano a través, por una parte, de la educación y, por otra, del grupo y las relaciones sociales (White, 1988).

Históricamente, el sistema educativo de Japón ha experimentado tres grandes reformas. La primera consistió en la apertura de la isla al mundo en 1868, apertura guiada por los imperativos del mundo occidental. El Japón Meiji de ese momento importó los planes administrativos del sistema educativo francés, la idea de las universidades nacionales alemanas, el modelo inglés de las escuelas basadas en principios morales y el paradigma pedagógico americano de John Dewey sobre que la escuela debe ser responsable del desarrollo de la persona (OCDE, 2012). En esta reforma se abolieron las distinciones de clase existentes para hacer la educación accesible a todos los ciudadanos. Este es el origen del que hoy se considera uno de los sistemas educativos más meritocráticos del mundo (OCDE, 2012).

La segunda gran reforma educativa tuvo lugar tras la Segunda Guerra Mundial, en 1947, con la aprobación de la nueva Constitución y la Ley Básica de Educación. Estas leyes establecieron el derecho de todo ciudadano japonés a la educación obligatoria durante nueve años. Antes de esta segunda reforma, los institutos y las instituciones de educación terciaria se diferenciaban por ámbito educativo, y el acceso a la universidad se limitaba a unos pocos estudiantes provenientes de ciertos institutos. Con la nueva ley, todos los institutos se dividieron en «baja» $y$ «alta» educación secundaria con igualdad de acceso para hombres y mujeres (single-tracked education system).

Por último, la tercera reforma educativa tuvo lugar en los años ochenta, con el objetivo de dar respuesta a los cambios económicos y sociales (aumento de la esperanza de vida, descenso de la natalidad, incremento de la población que accede a la universidad, disminución del sector primario de la economía y aumento de los sectores secundario y terciario, desregulación, privatización, reformas laborales y económicas liberales para mantener la competitividad), por lo que se impuso 
una agenda de reforma liberal que responsabilizaba al individuo de su aprendizaje a lo largo de la vida para tener éxito en la denominada nueva sociedad de riesgo. Esta reforma implicó un incremento de la oferta educativa, la privatización de la educación y un sistema más selectivo y competitivo, no solo entre los estudiantes, sino también entre las instituciones de oferta educativa. En línea con esta reforma de agenda liberal, en 1996 el Ministerio empezó a aplicar a una nueva filosofía educativa basada en adaptar la educación a las necesidades individuales e incrementar la capacidad de los estudiantes para actuar autónomamente y pensar creativamente. Para ello, redujo un $30 \%$ los contenidos del currículo obligatorio y dio plena autonomía a las escuelas y los institutos para adaptar los nuevos planes de estudio al proponer un nuevo rol para los profesores, que pasarían de ser meros diseminadores de conocimiento a ser coordinadores de proyectos para el estudio integrado, con el objetivo de que los estudiantes pudieran desarrollar competencias de creatividad, aprender autónomamente y solucionar problemas. Las fuertes críticas a esta reforma educativa propiciaron que en 2011 se tomaran nuevas medidas para un retorno al currículo prescriptivo y tradicional, y una nueva agenda educativa más conservadora, aunque los resultados tras la última reforma evidenciaban una mejora de la motivación de los estudiantes, de la relación estudiante-profesor y el clima del aula, y de las habilidades cognitivas de orden superior, que son las requeridas en la economía del conocimiento.

Actualmente, el sistema educativo de Japón se reconoce como uno de los más exitosos del mundo, no solo por su éxito académico o por los resultados de aprendizaje obtenidos en los informes PISA (OCDE, 2012), sino también por el ajuste entre las habilidades formadas y las habilidades requeridas por el mercado laboral, es decir, por la cualificación de la fuerza productiva de trabajo (CIEB, 2015), por lo que ha sido referente para el sistema educativo de muchos otros países. Esto es aún más admirable por el hecho de que su gasto en educación, público y privado, está por debajo de la media de los países de la OCDE en términos de porcentaje sobre el PIB del país (OCDE, 2012). Además, el sistema es altamente igualitario, tal y como muestra el hecho de que solo el $9 \%$ de la variación en el desempeño académico se explica por el origen socioeconómico, frente a la media del $14 \%$ de los países de la OCDE. La tasa de finalización de la educación secundaria es del $95 \%$. Además, su nivel de innovación, según el Global innovation index (Universidad de Cornell, INSEAD y OMPI, 2015) es alto, lo que lo sitúa en la posición 19, entre los 25 primeros países de los 141 que constan en este índice, aunque está detrás de Estados Unidos (5), Corea del Sur (14) y los países escandinavos — Suecia (3), Finlandia (6) y Dinamarca (10)—. Por último, la media de desempleo juvenil, con un 7,9 \% en 2012 (CIA, 2015) se sitúa por debajo de la media de los países de la OCDE, aunque va en aumento. 
El sistema educativo en Japón se inicia a los 6 años con la escuela primaria, cuya duración es de seis años, y luego continúa con tres años más en el instituto o en la lower secondary school. Así, se completan los nueve años de educación obligatoria establecida por el sistema. Tras esta, para poder acceder a la universidad, en las escuelas vocacionales o los junior college, deben completarse tres años más en un upper secondary school, por lo que se equipara la edad de inicio a la higher education con el resto de países.

Las opciones para poder acceder a la educación terciaria desde la educación secundaria son: (1) acceder a un junior college, dirigido principalmente a las mujeres, con una duración de dos o tres años, tras el cual puede accederse a la universidad; (2) acceder directamente a la universidad para cursar un bachelor degree de cuatro años; o (3) acceder a una escuela vocacional, que es el equivalente a la FP superior. Las dos primeras opciones proporcionan formación generalista, mientras que la tercera opción proporciona formación especialista (MEXT, 2015).

La educación preobligatoria (antes de los 6 años) recae en su mayor parte en organismos privados. En la educación primaria y secundaria, los padres pueden escoger libremente la escuela de sus hijos, y tras la educación obligatoria, las instituciones compiten entre ellas para reclutar estudiantes y obtener financiación. Esto ha hecho que las desigualdades sociales entre escuelas se hayan incrementado en las últimas décadas. En el nivel terciario, nuevamente vuelve a predominar el sector privado, con dos tercios de la educación terciaria financiada por este sector, lo que propicia que se alcancen los niveles de Estados Unidos, que es el primer país de la OCDE en cuanto a financiación privada. La competitividad para entrar en las mejores universidades públicas es tan alta que las familias destinan grandes sumas de dinero a pagar los centros privados que preparan para los exámenes de acceso, los denominados jukus (OCDE, 2012), durante la educación primaria y secundaria.

\section{Figura 4. Esauema del sistema educativo iavonés.}

Estudios de doctorado

Estudios de máster

Educación universitaria en el ámbito común o en FP

Educación secundaria superior (hasta los 18 años)

Educación secundaria júnior obligatoria (hasta los 15 años)

Educación elemental obligatoria (hasta los 12 años)

Fuente: Esquema adaptado del Ministry of Education, Culture, Sports, Science and Technology (MEXT) (2015). 
Aunque el sistema educativo de Japón ha sido tradicionalmente descrito como altamente centralizado, la realidad muestra que su centralización únicamente se produce en la financiación, mientras que otros aspectos, como la selección y evaluación de los profesores y la apertura de centros educativos, están altamente descentralizados, puesto que se encargan de ellos las 47 prefecturas que componen Japón; otro tanto ocurre con la selección de los libros de texto, cedida a las municipalidades. Las escuelas y los maestros tienen una gran libertad para escoger el método con el que formar para que se logren los estándares requeridos (OCDE, 2012). En síntesis, por tanto, es un sistema centralizado en la gestión de los recursos, pero descentralizado en el contenido educativo y las políticas de los centros educativos.

Japón se caracteriza por poseer un sistema educativo con bajos niveles de diferenciación vertical, es decir, que todos los estudiantes inician la educación obligatoria con la misma edad y no hay posibilidad de repetir, por lo que todos alcanzan el mismo nivel a los 15 años. Es en esta edad donde se da la primera selección, ya que existe la opción de dos itinerarios distintos.

La calidad de la formación profesional en Japón ha sido considerada uno de los fundamentos responsables del rápido crecimiento económico de Japón desde la Segunda Guerra Mundial y, en particular, del buen desarrollo de las habilidades que se requieren en el mercado de trabajo (Goodman, Hatakenanka y Kim, 2009).

Antes de la Segunda Guerra Mundial, Japón ofrecía dos itinerarios educativos en educación secundaria para acceder a la educación terciaria: uno que conducía a la universidad y otro que conducía a la FP. Bajo este sistema, las universidades no recibían financiación pública, mientras que las instituciones que proporcionaban FP, los senmongako, estaban totalmente financiados por el Gobierno (Yamamoto, 1997).

Tras la guerra, Japón cambió este sistema educativo de doble itinerario a una única vía o itinerario común, de modo que la FP perdió todo su estatus, ya que todos los estudiantes competían por entrar en la universidad y aquellos que no lo lograban, accedían a la FP o a la specialised higher education a través de los senmongako y los junior college. La diferencia entre los senmongako y los junior college es que mientras los primeros se concentran en la formación de profesionales o semiprofesionales, los segundos desarrollan las habilidades domésticas. En este sentido, los primeros tienen un equilibrio entre géneros, mientras que a los segundos solo acuden mujeres. Otra diferencia es que los primeros son libres de la intervención del Gobierno en el diseño de sus programas educativos, mientras que los segundos no (Goodman, Hatakenanka y Kim, 2009). 
Actualmente, no obstante, el $25 \%$ de los estudiantes acuden a los senmongako, donde tras obtener un diploma en dos o tres años, pueden optar a la universidad, o bien continuar dos años más de FP y obtener un diploma advanced. Aunque las escuelas especialistas de FP o specialised higher education mantienen en su currículo materias genéricas, como lengua japonesa, matemáticas y lengua extranjera, de un $30 \%$ a un $80 \%$ de los créditos se dedican a materias vocacionales. Desde 1994, sus formadores pueden ser reclutados de la industria.

El incremento de estudiantes en estos centros se explica porque esta opción de educación terciaria responde mejor a las demandas del sector empresarial y, por tanto, ofrece mayores posibilidades de empleo.

Las escuelas especialistas de FP se clasifican de acuerdo con ocho categorías laborales: ingeniería, gestión comercial, medicina, sanidad, artes liberales, educación y trabajo social, ciencia doméstica y agricultura.

La característica en común de estas instituciones es que están conectadas con el mercado de trabajo, de modo que la formación está orientada al trabajo y a la empleabilidad, y su objetivo es preparar para una cualificación vocacional.

Un caso especial de este tipo de centros, que han sido capaces de adaptarse con éxito a las demandas del contexto empresarial, son los institutos kogen, un caso particular de FP. Solo un $1 \%$ de los estudiantes que dejan secundaria entran a los 15 años en los institutos kogen, donde aprenden una profesión durante cinco años. Existen 57 institutos nacionales de tecnología en Japón, los llamados institutos kogen. Estos institutos tratan de cubrir la brecha de habilidades -o skills gap - entre la demanda de la industria y la oferta de las universidades, sobre todo en los campos científico y tecnológico. Forman en el entorno laboral e incluyen la participación de la industria local en el diseño de un currículo que se actualiza constantemente, lo que se denomina en otros países formación dual.

Japón es un buen ejemplo de cómo el sistema educativo puede integrarse con la industria a través de la FP. En este sentido, es muy diferente de otros países asiáticos, como, por ejemplo, Corea del Sur, donde la intervención del Estado sobre la educación terciaria es mucho mayor que en el caso de Japón, que deja la educación terciaria en su mayor parte en manos de las iniciativas privadas en un mercado liberalizado. Este hecho, unido al descenso de la natalidad en Japón, provoca que haya una competencia muy alta entre estas instituciones para captar estudiantes (Goodman, Hatakenanka y Kim, 2009).

Mientras que en Corea del Sur las instituciones de FP las gestiona el Estado, en Japón los senmongako son privados y autónomos, se orientan a las necesidades del mercado y hacen hincapié en la empleabilidad y la competitividad. En este caso, la industria y los empresarios han sido más activos que el Gobierno en la definición de los programas formativos de FP. Tradicionalmente, Japón se ha desta- 
cado por la alta implicación de sus empresas en la formación de sus trabajadores. Del mismo modo, esas empresas tradicionalmente se han implicado en el diseño de los currículos formativos de FP (Goodman, Hatakenanka y Kim, 2009). Así pues, las características definitorias del modelo de FP japonés son el estrecho vínculo con la industria, el alto compromiso de esta con la formación, y la gestión y financiación privadas de los centros que proporcionan este tipo de formación.

Los ministerios de Educación y de Trabajo interactúan para lograr un enfoque integrado de la FP, aunque quien toma el liderazgo es el Ministerio de Trabajo, que proporciona financiación a los gobiernos de las prefecturas y locales para la formación vocacional. El dinero proviene de un seguro de desempleo que las empresas pagan al Gobierno para los costes de formación. En síntesis, este modelo de FP se caracteriza por unas relaciones muy integradas entre las empresas y el sistema educativo (Velde, 2009).

\section{Similitudes y diferencias entre los sistemas de FP de los países de Extremo Oriente}

En Extremo Oriente la mayor parte de los países comparten su percepción en cuanto al enfoque que debe tener la FP para contribuir a las necesidades del mercado laboral y a las demandas sociales que caracterizan los últimos tiempos. Las principales diferencias se observan entre países que atraviesan etapas distintas en cuanto a su desarrollo económico. Para realizar la comparación de los sistemas de formación profesional en esta región, se hará hincapié en los siguientes aspectos: el marco político, institucional y legal, los mecanismos de financiación, la estructura, la calidad y la relevancia para cubrir las necesidades del mercado de trabajo.

En primer lugar, en lo que respecta al marco político, institucional y legal, la mayor parte de los países en Extremo Oriente tienen un marco legislativo sólido que asiste al modelo a través del cual se configura la FP. La legislación en materia de FP suele estar alineada con la que se aplica a otros niveles que implican la educación, la economía y el mercado de trabajo.

Los gobiernos o las entidades públicas, en un ámbito centralizado o más local, tienen buena parte de la responsabilidad a la hora de gestionar la FP. Sin embargo, cada vez es más importante la implicación de las empresas y de otros agentes sociales en el nivel terciario de la educación, lo que da lugar a diferencias entre los países analizados. Así, mientras en China y Corea del Sur, los ministerios que participan centralizadamente son los ministerios de Trabajo y Educación, en Taiwán, solo participa este último. El nivel de descentralización en cuanto a la organización de la FP también presenta una gran disparidad entre países. 
Por lo que respecta a la financiación, cada país utiliza su propio sistema. La financiación proviene de vías tanto públicas como privadas, lo que favorece grandes diferencias entre países a la hora de intervenir el Estado en el diseño y la gestión de la FP, y en la autonomía de los centros que imparten esta formación. En China, por ejemplo, tradicionalmente los gobiernos a nivel tanto central como local invierten poco en este tipo de formación, puesto que son las tasas, tuition fees, las que contribuyen de forma importante a la financiación del sistema. Esta situación ha cambiado en los últimos años, en los que se han introducido ayudas y exenciones para estudiantes con mayores dificultades, por ejemplo, para los que proceden del ámbito rural y tienen menos posibilidades de acceder a este tipo de formación (Unesco, 2011). Taiwán también ha evolucionado mucho en los últimos años en este mismo sentido con la intención de proporcionar este tipo de formación de forma gratuita y generalizada, y con el aumento de la exención del pago de tasas y ayudas financieras para los estudiantes con mayores necesidades (Ministry of Education, 2014). En el caso de Corea del Sur, por un lado, la FP se financia a través del presupuesto ordinario del Ministerio de Educación y, por otro lado, en los canales no formales, se recaudan tasas por parte de los empleadores cuando contratan, que se les reembolsan cuando imparten la formación a sus trabajadores. Este tipo de tasas constituyen la fuente más importante de recursos en los programas no formales de formación en habilidades, incluidos la formación a desempleados, el aprendizaje autodirigido de empleados y los programas de aprendizaje dirigidos por el empleador. El Gobierno coreano también está considerando estas vías de financiación para que puedan aplicarse a la FP formal. En ocasiones, en esta región también se aplican sistemas de financiación basados en resultados. Un buen ejemplo es, de nuevo, Corea del Sur, donde el Gobierno selecciona un número de escuelas de FP que tienen importancia estratégica, las denominadas meister high schools, y les proporciona financiación para formar a los estudiantes en las competencias más avanzadas y actualizadas. En síntesis, en relación con los niveles de intervención del Estado en la FP y de financiación pública de los centros, podría establecerse el siguiente orden de mayor a menor nivel de intervención en los países analizados: China, Corea del Sur, Taiwán y Japón.

Otro aspecto importante que debe analizarse es la estructura que caracteriza los modelos de FP y cómo se integran en el contexto educativo general. En resumen, la FP se divide en dos grandes categorías, lo que podría denominarse la FP inicial y la formación continua, que se integra en lo que se conoce como aprendizaje a lo largo de la vida. Los centros involucrados en la FP son escuelas, colleges de FP y centros de formación, aunque también se utiliza la formación en el lugar de trabajo (on-the-job) en los itinerarios tanto formales como no formales de FP. Los 
estudios de FP también se imparten en distintos niveles, que pueden encontrase en los niveles de secundaria y en los estudios de tercer nivel o universitarios. A pesar de esta estructura común en todos los países analizados, existen algunas diferencias que requieren una mención especial, en particular, en lo que respecta a los años de educación obligatoria. En este sentido, China se diferencia del resto de países analizados, puesto que el periodo de educación obligatoria es inferior. Consecuentemente, los estudiantes que optan por la vía profesional inician esta trayectoria a una edad más temprana (12 años, respecto a los 15 años del resto de países).

Cabe mencionar también el uso de aprendices o la formación dual para adquirir las habilidades que demanda el mercado de trabajo. En muchos casos, los estudiantes en la región analizada toman parte en actividades de formación y entrenamiento uno o dos días a la semana, y durante el resto de la semana se supervisa su trabajo. También se usa la fórmula de organizar el entrenamiento en bloques y que se supervise su trabajo el resto del tiempo. Los contratos formales entre empleadores, organizaciones de formación o entrenamiento y los estudiantes son bastante comunes. En el caso específico de Japón, los programas de entrenamiento dual se implementan principalmente por parte de las instituciones de formación o educación, que dependen del Gobierno o de la prefectura. Además, la formación en el lugar de trabajo se ofrece durante un tiempo prefijado y consiste en coger a un estudiante no entrenado y combinar el entrenamiento en el lugar de trabajo y la formación en la institución formativa correspondiente. En China, por el contrario, el uso de aprendices se utiliza de manera no oficial, por lo que no hay una regulación reseñable al respecto.

En relación con la calidad y la proximidad de la FP al mercado de trabajo, hay que decir que en los últimos años se han producido una serie de reformas curriculares que afectan a la FP en el contexto asiático, y que han hecho hincapié en el aprendizaje basado en competencias. Con esto se pretende fortalecer la respuesta del sistema formativo en el ámbito de la FP a las necesidades de la industria y el tejido productivo. Japón y Corea del Sur son dos ejemplos de países que han instaurado este aprendizaje basado en competencias y que destacan respecto a los otros dos países analizados, China y Taiwán. De igual manera, para asegurar la calidad, estos dos países, junto con Taiwán, han tomado la delantera al resto de países analizados en la introducción de un sistema de acreditación o certificación de su modelo de FP, que hace referencia al proceso para asegurar que los centros que proporcionan entrenamiento y formación tengan la capacidad para hacerlo y, a su vez, cumplan unos estándares de calidad. 


\section{Conclusiones}

Como último apartado de este trabajo se incluye una enumeración de los principales puntos fuertes de los modelos educativos que se desarrollan en la región analizada, con especial mención a los modelos de FP, lo que permitirá apreciar mejor los aciertos de los modelos aplicados a los distintos países analizados, y extraer lecciones que puedan extrapolarse a otras regiones. Muchos de estos aciertos se determinan en comparación con otros países; y otros, en relación con la evolución del modelo educativo del propio país con el paso del tiempo.

Tabla 2. Puntos fuertes de los modelos educativos en Extremo Oriente.

\begin{tabular}{|c|c|c|c|}
\hline China & Taiwán & Corea del Sur & Japón \\
\hline $\begin{array}{l}\text { - Establecimiento } \\
\text { de nueve años de } \\
\text { escolarización } \\
\text { obligatoria. } \\
\text { - Número creciente de } \\
\text { estudiantes que optan } \\
\text { por la formación } \\
\text { secundaria superior y } \\
\text { universitaria. } \\
\text { Modelo de FP de } \\
\text { nivel superior que } \\
\text { involucra todo } \\
\text { un conjunto de } \\
\text { especialidades, } \\
\text { habilidades en } \\
\text { los programas } \\
\text { formativos, la } \\
\text { formación en } \\
\text { empresas y la } \\
\text { colaboración entre el } \\
\text { sistema educativo y la } \\
\text { industria. } \\
\text { Ayudas } \\
\text { gubernamentales } \\
\text { para financiar la } \\
\text { educación secundaria } \\
\text { superior. } \\
\text { El profesorado de FP } \\
\text { debe pasar un mes } \\
\text { al año o dos meses } \\
\text { cada dos años en la } \\
\text { industria. También } \\
\text { es frecuente la figura } \\
\text { del profesor a tiempo } \\
\text { parcial. }\end{array}$ & $\begin{array}{l}\text { - Excelente } \\
\text { cooperación entre } \\
\text { industria y academia. } \\
\text { - Formación en } \\
\text { habilidades prácticas, } \\
\text { learning by doing, } \\
\text { certificación } \\
\text { profesional tanto } \\
\text { para estudiantes } \\
\text { como para profesores. } \\
\text { - Fortalecimiento de la } \\
\text { enseñanza práctica en } \\
\text { los centros de FP. } \\
\text { Implicación de } \\
\text { recursos de la } \\
\text { industria para } \\
\text { la enseñanza } \\
\text { colaborativa en FP. } \\
\text { Motivación de } \\
\text { la participación } \\
\text { competitiva de los } \\
\text { estudiantes. } \\
\text { Cooperación entre } \\
\text { la academia y la } \\
\text { industria en términos } \\
\text { de innovación e I+D. } \\
\text { Estudiantes } \\
\text { internacionales } \\
\text { y cooperación } \\
\text { internacional. }\end{array}$ & $\begin{array}{l}\text { - Alto porcentaje, el } \\
97 \% \text { de los jóvenes } \\
\text { de entre } 25 \text { y } 34 \\
\text { años, en formación } \\
\text { secundaria superior, y } \\
\text { un } 53 \% \text { en educación } \\
\text { universitaria o de } \\
\text { tercer nivel. } \\
\text { - Buena valoración de } \\
\text { la educación por toda } \\
\text { la sociedad coreana } \\
\text { y aspiraciones } \\
\text { formativas muy } \\
\text { elevadas de los } \\
\text { estudiantes. } \\
\text { Resultados del } \\
\text { informe PISA } \\
\text { excelentes. } \\
\text { Implicación del tejido } \\
\text { productivo, empresas } \\
\text { y organizaciones en } \\
\text { las políticas de FP. } \\
\text { Mejora de la FP de } \\
\text { tercer nivel, que se } \\
\text { desarrolla en los } \\
\text { colleges, en el ámbito } \\
\text { universitario. } \\
\text { Buena base de } \\
\text { investigación en FP } \\
\text { de tercer nivel. } \\
\text { En principio, buena } \\
\text { articulación del } \\
\text { sistema educativo } \\
\text { de tercer nivel, lo } \\
\text { que les permite a los } \\
\text { estudiantes continuar } \\
\text { programas de FP } \\
\text { de tercer nivel hacia } \\
\text { grados universitario. }\end{array}$ & $\begin{array}{l}\text { - Eficiencia del sistema } \\
\text { educativo. } \\
\text { - Alto compromiso } \\
\text { del Gobierno y la } \\
\text { sociedad con la } \\
\text { educación. } \\
\text { - Alto compromiso de } \\
\text { la industria japonesa } \\
\text { con la formación. } \\
\text { - Sistema igualitario y } \\
\text { meritocrático. } \\
\text { - Sistema basado en } \\
\text { valores sociales. } \\
\text { - Alta cualificación de } \\
\text { los profesores y alto } \\
\text { estatus asociado a la } \\
\text { profesión. }\end{array}$ \\
\hline
\end{tabular}

Fuente: Elaboración propia. 
Como cabe apreciar en la tabla 2, los aciertos más destacados en los modelos educativos en el área analizada podrían resumirse en la generalización y universalización de la educación, lo que está permitiendo contar con una fuerza de trabajo formada en distintos niveles, de lo que se beneficia el tejido productivo. Por otra parte, en lo que se refiere a la FP, su integración en el sistema educativo general está muy bien articulada, lo que permite un desarrollo educativo y profesional del estudiante hasta llegar a los niveles más avanzados, y pueden seguirse distintos itinerarios que incluyen también la FP. La orientación de los estudios de FP, aunque no de forma exclusiva, se centra en la formación competencial, donde lo importante no es solo que el estudiante adquiera conocimientos, sino también que desarrolle un perfil competencial que lo cualifique ante las demandas del mercado laboral (Fitó-Bertrán, Hernández-Lara y Serradell-López, 2014 y 2015; Hernández-Lara, Gorjup y Cascón, 2010). Para ello, es fundamental que se impliquen de forma destacada la industria, el tejido productivo y las empresas, que deben expresar sus demandas en cuanto a las habilidades y competencias necesarias en su personal. De esta manera, se tendrá una guía útil que dirija el desarrollo curricular de los estudiantes. Esta involucración de la industria y las empresas puede articularse de varias maneras, que implican un determinado papel más o menos activo por parte del tejido productivo y empresarial, ya sea al determinar de manera más pasiva las competencias necesarias o al influir de forma más activa en su transmisión y adquisición, ya sea al acoger al profesorado y permitir su reciclaje, o al acoger al alumnado y permitir su formación práctica y experiencial. Por lo que respecta al posicionamiento de la FP dentro del modelo educativo, cabe destacar el desarrollo de este tipo de formación en distintos niveles, que incluso alcanzan niveles superiores o universitarios, hasta llegar al ámbito de la investigación en el caso de algunos modelos. Esto implica un desarrollo competencial mucho más sofisticado, sobre todo en lo que se refiere a las competencias innovadoras y creativas, con la importancia que esto tiene para atender a las demandas del tejido productivo, incluidas tanto las grandes corporaciones como las pequeñas y medianas empresas, lo que, además, está sirviendo para aumentar la reputación de este tipo de formación y mejorar la captación de estudiantes. 


\section{Bibliografía}

Agencia Central de Inteligencia (CIA) (año): The world factbook, en línea. $<$ www.cia.gov/library/publications/the-world-factbook/fields/2229.html >. (Consultado el 12/10/2015).

Banco Mundial (2015): World development indicators.

Center on International Education Benchmarking (CIEB) (año): Japan: school-to-work transition, en línea. <www.ncee.org/programs-affiliates/centeron-international-education-benchmarking/top-performing-countries/japanoverview/japan-school-to-work-transition >. (Consultado el 12/10/2015).

Chang, S. J. (2009): «A cultural and philosophical perspective on Korea's education reform: a critical way to maintain Korea's economic momentum», Academic Paper Series on Korea, Washington: Korea Economic Institute.

Comisión Económica y Social de las Naciones Unidas para Asia y el Pacífico (CESPAP) (2014): Statistical yearbook for Asia and the Pacific 2014, Bangkok: CESPAP.

Education System Taiwan (2011): The Taiwanese education system described and compared with the Dutch system, vol. 3, segunda edición, EP Nuffic, Internationalizing Education.

Fitó-Bertrán, A.; Hernández-Lara, A. B.; y Serradell-López, E. (2014): «Comparing student competences in a face-to-face and online business game», Computers in Human Behavior, 30, pp. 452-459.

- (2015): «The effect of competences on learning results: an educational experience with a business simulator», Computers in Human Behavior, 51, pp. 910-914.

Fondo de Cooperación y Desarrollo Internacional (ICDF) (2010): «Special report», Vocational education. Extending Taiwan's vocational education system to Central America, Taipéi: International Cooperation and Development Fund.

Goodman, R.; Hatakenanka, S.; y Kim, T. (2009): «The changing status of vocational higher education in contemporary Japan and the Republic of Korea», Unesco-Unevoc Discussion Paper Series, Bonn: Unesco-Unevoc International Center for Technical and Vocational Education and Training.

Harden, B. (2011):«With workplace training, Japan's Kosen colleges bridge 'skills gap'», The Hechinger Report, en línea. <hechingerreport.org/withworkplace-training-japans-kosen-colleges-bridge-skills-gap >. (Consultado el 13/10/2015).

Hernández-Lara, A. B.; Gorjup, M. T.; y Cascón, R. (2010): «The role of the instructor in business games: a comparison of face-to-face and online 
instruction», International Journal of Training and Development, 14 (3), pp. 169-179.

Hwang, S. (2007): Shift in skills structure in Korea and core technical workforce, Corea: Labor Institute.

Jones, R.S. (2013): «Education reform in Korea», OECD Economics Department Working Papers, 1067, OECD Publishing.

Kuczera, M.; Kis, V; y Wurzburg, G. (2009): A learning for jobs review of Korea. OECD reviews of vocational education and training, París: OECD.

Ministerio de Educación (1996): Vocational education law of the people's Republic of China, Pekín: MoE.

Ministry of Education (2012): Technological $\mathcal{E}$ vocational education in Taiwan, ROC, Taipéi: MoE.

- (2014): Technological E vocational education in Taiwan, ROC, Taipéi: MoE.

Organización para la Cooperación y el Desarrollo Económicos (OCDE) (2010): Learning for jobs. OECD reviews of vocational education and training, París: OECD.

- (2012): Lessons from PISA for Japan, strong performers and successful reformers in rducation, OECD Publishing.

- (2015): Economic review of China, París: OECD.

PARK, D. Y. (2011)ः «Korean policies on secondary vocational educationः efforts to overcome mismatch and labor force shortage», $B I B B-B W P, 3$.

The Republic of China yearbook 2014 (2014): En línea: <issuu.com/eyroc/docs/ rocyearbook2014>. (Consultado el 15/04/2016).

Unesco (2011): Asia-Pacific regional background paper for the third international congress on TVET, Bangkok: Unesco.

UnEsCo-IBE (2011): World data on education: people's Republic of China. vii edición, 2010-2011, Ginebra: Unesco-IBE.

Unesco-Unevoc (2013): World TVET database China, international centre for technical and vocational education and training, Bonn: Unesco-Unevoc.

Universidad de Cornell, INSEAD y Organización Mundial de la Propiedad Intelectual (OMPI) (2015): «The global innovation index: effective innovation policies for development», World Intellectual Property Organisation, Ginebra. ISSN 2263-3693.

Velde, C. (2009): «Exploring opportunities for collaborative linkages in the vocational education and training (VET) sector: a comparative study of VET in Australia and Japan», en C. VELDE (ed.): International perspectives on competences in the workplace, Adelaida: Springer, pp. 21-32. 
White, M. (1988): The Japanese education challenge: a commitment to children, Nueva York: The Free Press.

ҮАмамото, S. (1997): «The role of Japanese higher education system in relation to industry», en G. Akira y O. Hirokyuki (eds.): Innovation in Japan, Oxford: The Clarendon Press, pp. 294-307. 